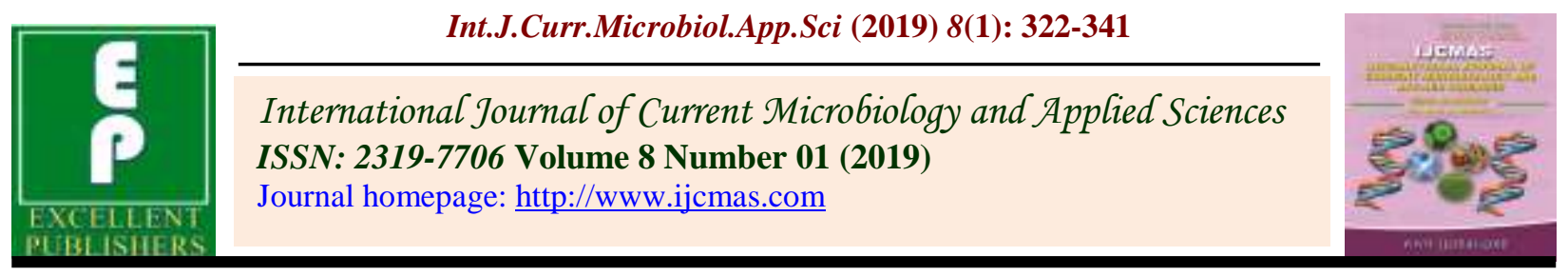

Original Research Article https://doi.org/10.20546/ijcmas.2019.801.034

\title{
Relative Efficiency of Udaipur Rock Phosphate Combined with Amendments in Acid Soils of Odisha, India
}

\author{
Debasis Sarangi*, Dinabandhu Jena and Kabita Mishra \\ Orissa University of Agriculture and Technology, Bhubaneswar-751003, Odisha, India \\ *Corresponding author
}

\begin{tabular}{|l|}
\hline Ke y w o r d s \\
Hybrid napier \\
grass, Udaipur rock \\
phosphate, SSP, \\
acid soils, Lime, \\
PSB, Farmyard \\
manure, Biomass, \\
Available P, Exch. \\
Ca
\end{tabular}

\section{A B S T R A C T}

\begin{abstract}
A pot culture study was conducted during February, 2014 to evaluate the effect of organic manure, PSB or lime on Udaipur Rock Phosphate (URP) dissolution, $\mathrm{P}$ and Ca availability and biomass yield of hybrid napier grass in three different acid soils (Typic Halpludalf) in Odisha, India. The experiment was conducted in a completely randomized design (CRD) with three replications and 18 treatments consists of 3 low $\mathrm{pH}$ soils $-\mathrm{S}_{1}(\mathrm{pH}-4.15), \mathrm{S}_{2}(\mathrm{pH}-$ 5.03), $\mathrm{S}_{3}(\mathrm{pH}-5.82)$ and six rock phosphate treatments $-\mathrm{T}_{1}$-Control, $\mathrm{T}_{2}-200 \% \mathrm{P}$ through URP, $\mathrm{T}_{3}-50 \% \mathrm{P}$ through URP+50\%P through $\mathrm{SSP}, \mathrm{T}_{4^{-}} 100 \% \mathrm{P}$ through URP +FYM @ 5 tha $^{-1}, \mathrm{~T}_{5}-100 \% \mathrm{P}$ through URP +PSB @ $10 \mathrm{~kg} \mathrm{ha}^{-1}$ and $\mathrm{T}_{6}-100 \% \mathrm{P}$ through URP + lime @ 0.2 LR (Lime Requirement). The URP namely sourced from FCI Aravali Gypsum and Minerals India Limited (FAGMIL), Jodhpur contains $7.8 \%$ total P, $25.6 \% \mathrm{Ca}, 0.26 \% \mathrm{Mg}$ and $0.24 \% \mathrm{~K}$ indicating a moderate reactive material. Application of URP alone or with amendments increased soil $\mathrm{pH}$ significantly, attained its peak at $4^{\text {th }}$ cutting and then decreased gradually, but remained above the initial value at the end of $8^{\text {th }}$ cutting. Among the treatments, URP + lime $\left(\mathrm{T}_{6}\right)$ recorded highest $\mathrm{pH}$ value followed by $200 \% \mathrm{URP}\left(\mathrm{T}_{2}\right)$ and URP $+\operatorname{SSP}\left(\mathrm{T}_{3}\right)$ in all soils. Available $\mathrm{P}$ in control decreased gradually during the growth period. In other treatments, $P$ content increased and attained its peak at $2^{\text {nd }}$ cutting, there after declined but remained above the initial value at the end of $8^{\text {th }}$ cutting irrespective of the soils. P build up in sole URP (200\% P) treatment was maximum (11 $\left.14.5 \mathrm{~kg} \mathrm{ha}^{-1}\right)$ followed by URP +SSP $\left(8.9-12.3 \mathrm{~kg} \mathrm{ha}^{-1}\right)$ and URP + lime (6.8-9.0 kg ha $\left.{ }^{-1}\right)$. Exchangeable calcium content in control is decreased by $52-58 \%$ over the initial value due to crop removal. Combined application of URP + SSP recorded highest exchangeable calcium content followed by URP + lime and URP alone. Sole application of URP recorded highest biomass yield in $\mathrm{S}_{1}(44 \%)$ and $\mathrm{S}_{2}(41 \%)$ whereas, URP+SSP recorded highest yield in $\mathrm{S}_{3}(47 \%)$ might be due to the dissolution of URP got slower with increased in soil $\mathrm{pH}\left(\mathrm{S}_{3}\right)$. The relative agronomic effectiveness (RAE) of URP was higher when it was applied at higher dose $\left(\mathrm{T}_{2}\right)$ in low pH soil viz. $\mathrm{S}_{1}(107 \%)$ and $\mathrm{S}_{2}(108 \%)$ but the efficiency decreased in $\mathrm{S}_{3}(76 \%)$. The efficiency of URP is greatly influenced by soil $\mathrm{pH}$ and exchangeable calcium content of soils.
\end{abstract}

\section{Introduction}

Acid soils in India occupy about 90 million ha (Mha) out of which 49 Mha have $\mathrm{pH}$ less than
5.5. The supply of soil phosphorus has been a major limiting factor in crop production due to high $P$ fixation when a water soluble phosphate fertilizer is added to soil, a series of 
chemical reaction may take place. The dissolved $\mathrm{P}$ reacts with dissolved $\mathrm{Ca}$ (in high $\mathrm{pH}$ soils) or dissolved $\mathrm{Fe}$ and $\mathrm{Al}$ (in low $\mathrm{pH}$ soils) becomes more stable, forming precipitation with $\mathrm{Fe}, \mathrm{Al}, \mathrm{Ca}$ that are less available to plants (Barrow, 1983). In acid soils much of $\mathrm{P}$ is adsorbed by reacting with $\mathrm{Fe}, \mathrm{Al}$ and clay minerals or $\mathrm{Al}$ that is associated with organic matter (Huges and Gilkes, 1994). All these reactions can resulted in decreasing $\mathrm{P}$ availability over time (Hedley and McLaughlin, 2005; Syers et al., 2008). The direct use of phosphate rocks may be an economically viable alternative source of Pfertilizers in tropics. The developing countries like India can save huge amount of foreign exchange if phosphate rock (PR) can be used alone or with $\mathrm{P}$-fertilizer in acid soils.

The PR deposits in India including all grades and types is of 260 million tonnes out of which 15.27 million tonnes of high grade. The low grade $\mathrm{PR}$ is unacceptable to $\mathrm{P}$-fertilizer industry due to its low $\mathrm{P}_{2} \mathrm{O}_{5}$ and high $\mathrm{CaCO}_{3}$ content. This low grade PR could be a cheaper $P$ source for small and marginal farmers in acid soil regions. The efficiency of phosphate rock depends on its solubility which is influenced by chemical and mineralogical characteristics of rocks, soil properties, crops and climatic conditions (White, 1988b).The dissolution of phosphate rocks depend on the $\mathrm{H}^{+}$ion supply power of soils (Wheeler and Edmeades, 1984), activities of $\mathrm{Ca}^{2+}$ and $\mathrm{H}_{2} \mathrm{PO}_{4}{ }^{-}$ions in soil solution (Kirk and Nye, 1986b). Mishra and Pattanaik (1997), Pattanaik (1988), Dash et al., (1988) evaluated the efficiency of several Indian phosphate rocks with North Carolina, Gafsa, Florida, Morocco and found all the Indian phosphate rocks showed lower efficiency as compared to North Carolina with respect to yield and $\mathrm{P}$ availability.

Liming of acid soils is a common practice to raise soil $\mathrm{pH}$ and decrease $\mathrm{Al}$ toxicity for optimal crop growth. However, the higher $\mathrm{pH}$ and increased exchangeable $\mathrm{Ca}$ resulting from liming are detrimental to PR dissolution (Hammond et al., 1986b; Mishra and Pattanaik, 1997). Hence, lime rates should be carefully chosen to alleviate the Al toxicity problem and, at the same time, to avoid adverse effects on PR dissolution in acid soils (Chien and Friesen 1992). Application of phosphate solubilising biofertilizer (PSB) enhances dissolution of PR through production of organic acid and chelating substances (Sanyal and Saha, 1988; Adhya et al., 2015). Organic manures supplies plant nutrients such as $\mathrm{P}$ through decomposition and the organic acids produced in this process chelate P-fixing elements in the rhizosphere or decomposition system. Several studies showed that application of SSP and PR mixture in 1:1 ratio increased the dry matter yield and $\mathrm{P}, \mathrm{Ca}$ and $\mathrm{Mg}$ uptake by maize, groundnut, and linseed in acid soils (Mitra and Mishra, 1991; Das et al., 1990; Dwivedi and Dwivedi, 1990).

Although sizeable informations are available on rate of PR dissolution either alone or in combination with different amendments, such information is still lacking in published work dealing with direct use of Udaipur rock phosphate in acid soil region of Odisha, India.

In view of the above said knowledge gaps, a pot culture study was carried out to evaluate the effect of organic manure, PSB or lime on URP dissolution, $\mathrm{P}$ and $\mathrm{Ca}$ availability and biomass yield of hybrid napier grass in three different acidic laterite soils.

\section{Materials and Methods}

Three acidic laterite soil samples in bulk from plough layers $(0-15 \mathrm{~cm})$ were collected from farmer's field having maize-groundnut cropping system from Dhenkanal block of Dhenkanal district, Odisha. The collected soil samples were air dried, processed and used for 
pot culture experiment and laboratory analysis. Particle size was determined by Bouyoucos hydrometer method (Bouyoucos, 1962), pH by glass electrode with Calomel as standard (Jackson, 1973). Organic carbon was determined by wet digestion method of Walkley and Black (1934).The cation exchange capacity was determined by Schollenberger and Simon (1945). Nitrogen content in soil samples and organic manure was determined by Kjeldhal digestion method as described in AOAC (1995). Exchangeable $\mathrm{Ca}$ and $\mathrm{Mg}$ was determined by EDTA (Versenate) titration method (Gupta, 2007), exchangeable acidity and $\mathrm{Al}$ by the procedure outlined by McLean (1965). Available $\mathrm{N}$ in soils was determined by modified alkaline permanganate method (Subbiah and Asija, 1956), available P by Bray's 1 method (Bray and Kurtz, 1945) and available K by ammonium acetate method (Hanway and Heidel, 1952). The lime requirement value was determined by Woodruff Buffer method (Woodruff, 1948).

A pot culture experiment was carried out during February, 2014 in the green house of Department of Soil Science and Agricultural Chemistry, Orissa University of Agriculture and Technology, Bhubaneswar, Odisha. The experiment was conducted in a completely randomized design (CRD) with three replications and 18 treatments consists of 3 low $\mathrm{pH}$ soils $-\mathrm{S}_{1}(\mathrm{pH}-4.15), \mathrm{S}_{2}(\mathrm{pH}-5.03), \mathrm{S}_{3}$ (pH-5.82) and each soil was superimposed with six rock phosphate $(\mathrm{PR})$ treatments- $\mathrm{T}_{1^{-}}$ Control, $\mathrm{T}_{2}-200 \% \mathrm{P}$ through URP, $\mathrm{T}_{3}-50 \% \mathrm{P}$ through URP+50\%P through SSP, $\mathrm{T}_{4^{-}} 100 \% \mathrm{P}$ through URP +FYM @ 5 tha $^{-1}, \mathrm{~T}_{5}-100 \% \mathrm{P}$ through URP +PSB @ $10 \mathrm{~kg} \mathrm{ha}^{-1}$ and $\mathrm{T}_{6^{-}}$ 100\% P through URP + lime @ 0.2 LR (Lime Requirement).

The polyethylene lined earthen pots were rinsed in $0.1 \mathrm{~N} \mathrm{HCl}$ followed by deionised water. Seven $\mathrm{kg}$ of soil was transferred into each pot. Each pot received a common dose of $\mathrm{N} @ 40 \mathrm{~kg} \mathrm{ha}^{-1}$ through urea and $\mathrm{K}_{2} \mathrm{O} @ 40 \mathrm{~kg}$ $\mathrm{ha}^{-1}$ through mutate of potash. Phosphate @ 40kgP $\mathrm{O}_{5} \mathrm{ha}^{-1}$ was applied through Udaipur rock Phosphate or SSP as per the treatments. Well decomposed FYM was added @ 5tha ${ }^{-1}$ in $\mathrm{T}_{4}$. In $\mathrm{T}_{6}$, pure $\mathrm{CaCO}_{3}$ was added @ 0.2LR. The LR for different soil was: $\mathrm{S}_{1}-5.8 \mathrm{qha}^{-1}, \mathrm{~S}_{2^{-}}$ 4.8qha ${ }^{-1}$, and $\mathrm{S}_{3}-3.3 \mathrm{qha}^{-1}$. On soil weight basis, the fertilizers, FYM and PSB were calculated, mixed thoroughly with $7 \mathrm{~kg}$ of soil before planting. One slip of Bajra napier hybrid grass (Pennisetum glaucum) $\times$ (Pennisetum purpureum) was planted in each pot, watering with deionised water and plant protection measures were taken as and when necessary. The first cut was made after 60 days after planting and subsequently seven cuts were made at an interval of 45 days. Soil samples were collected from each treatments during cutting. After each cut, each pot received $\mathrm{N} @ 40 \mathrm{~kg} \mathrm{ha}^{-1}$ through urea solution. After recording the dry mass yield of grass at each cut, the samples were washed with acidified solution, rinsed with deionised water, dried at 65 degree centigrade in a hot air oven, grinded and kept for analysis. The dry powdered grass samples were digested with diacid mixture on a hot plate and filtered through Whatman No. 42 filter paper for estimation of $\mathrm{P}, \mathrm{Ca}$ and $\mathrm{S}$. The soil samples were air dried sieved through 8 mesh sieve and analysed for $\mathrm{pH}$, available $\mathrm{P}$ and exchangeable $\mathrm{Ca}$. Simple correlation was carried out to establish the relationships between biomass yield and soil properties.

\section{Results and Discussion}

\section{Characteristics of soil, rock phosphate and farmyard manure used in study}

The Alfisols used in this study were very acidic having $\mathrm{pH}: \mathrm{S}_{1}-4.15, \mathrm{~S}_{2}-5.03$ and $\mathrm{S}_{3}-$ 5.82. The soil texture varied from sandy loam to sandy clay loam. The soils had low to 
medium in organic carbon content, available $\mathrm{P}$ but low in available $\mathrm{N}$ and cation exchange capacity. Available $\mathrm{K}$ was medium to high (Table 1).

The samples of URP used namely sourced from FCI Aravali Gypsum and Minerals India Limited (FAGMIL), Jodhpur had 7.8\% total P, $25.6 \% \mathrm{Ca}, 0.26 \% \mathrm{Mg}$ and $0.24 \% \mathrm{~K}$, indicating a moderate reactivity of the material (Table 2).

The farmyard manure sample had $1.2 \% \mathrm{~N}$, $0.006 \% \mathrm{P}$ and $0.045 \% \mathrm{Ca}$ indicating a higher sink for $\mathrm{P}$ and $\mathrm{Ca}$ during dissolution of rock phosphate (Table 3).

\section{Effect of URP with SSP, FYM, PSB or lime on soil properties}

\section{Soil pH}

In all treatments soil $\mathrm{pH}$ increased significantly from its initial value, attained the peak at fourth cutting and then decreased gradually upto eighth cutting (Table 4 and Fig. 1 ). At the end of $8^{\text {th }}$ cutting, soil $\mathrm{pH}$ in control treatment attained its initial values or slightly higher in all soils whereas, in other treatments, it was higher than the in initial value, highest being in $\mathrm{T}_{2}$. On the other hand, combined application of URP+ lime@0.2LR recorded peak $\mathrm{pH}$ value at $3^{\text {rd }}$ cutting, the values were higher than all other treatments upto $6^{\text {th }}$ cutting. Addition of FYM or PSB with URP recorded lower $\mathrm{pH}$ value as compared to $\mathrm{URP}+\mathrm{SSP}\left(\mathrm{T}_{3}\right)$ treatment in all cuttings.

\section{Soil available phosphorus at different stages of cutting}

The available phosphorus content in control pot generally declined with progress of growth of hybrid napier grass. The magnitude of depletion was highest $\left(5.51 \mathrm{~kg} \mathrm{ha}^{-1}\right)$ in $\mathrm{S}_{3}(\mathrm{pH}-$ 5.82) followed by $3.83 \mathrm{~kg} \mathrm{ha}^{-1}$ in $\mathrm{S}_{2}$ (pH-5.03) and $2.71 \mathrm{~kg} \mathrm{ha}^{-1}$ in $\mathrm{S}_{1}(\mathrm{pH}-4.15)$ might be due to $\mathrm{P}$ uptake by grass (Table 5 and Fig. 2). The available $\mathrm{P}$ content in other treatments increased over the initial value, attained its peak at $2^{\text {nd }}$ cutting, there after declined but remained above the initial value at the end of $8^{\text {th }}$ cutting. This indicates that application of URP with SSP, FYM or PSB could meet crop requirement in long run. Sole application of URP at higher dose $(200 \%$ P) was better than $\mathrm{URP}+\mathrm{FYM}$ or URP+PSB treatment but can be compared with URP+SSP treatment in long run. Higher soil $\mathrm{P}$ content in $\mathrm{T}_{2}(200 \% \mathrm{P}$ through URP) treatment resulted in higher dissolution of URP in low $\mathrm{pH}$ soils varying from 4.15 to 5.82. Combined application of URP+SSP $\left(\mathrm{T}_{3}\right)$ seems to be better than URP+ lime treatment since, water soluble SSP meet the crop requirement $\mathrm{P}$ at initial stage and dissolution of URP build up the $\mathrm{P}$ status and also meet crop requirement in long run. On the other hand, inclusion of lime increased the soil $\mathrm{pH}$ that lower down dissolution rate of URP although calcium in lime decreases Al toxicity and helps better crop growth and biomass production. Inclusion of FYM with URP was better than URP+ PSB treatment. Since, FYM increases available $\mathrm{P}$ in soil through chelation and decomposition.

Available $\mathrm{P}$ build up in different treatments was calculated as final $\mathrm{P}$ minus initial $\mathrm{P}$. The data showed that irrespective of the soils, the $\mathrm{P}$ build in $\mathrm{T}_{2}(200 \% \mathrm{P}$ through URP) was highest followed by URP $+\operatorname{SSP}\left(\mathrm{T}_{3}\right)$ and URP $+\operatorname{lime}\left(\mathrm{T}_{6}\right)$.

\section{Soil exchangeable calcium at different stages of cutting}

During dissolution of rock phosphate, calcium is released and the soils with high calcium content would slow down the dissolution of rock phosphate. The acid alfisol used in this study had low exchangeable calcium varying from 1.32 to $1.50 \mathrm{c} \mathrm{mol}\left(\mathrm{P}^{+}\right) \mathrm{kg}^{-1}$ (Table 1). 
Table.1 Physical and chemical properties of the soil

\begin{tabular}{|c|c|c|c|c|c|c|c|c|c|c|c|c|c|c|c|}
\hline $\begin{array}{l}\text { Soil } \\
\text { type }\end{array}$ & $\begin{array}{c}\text { Sand } \\
(\%)\end{array}$ & $\begin{array}{l}\text { Silt } \\
(\%)\end{array}$ & $\begin{array}{l}\text { Clay } \\
(\%)\end{array}$ & $\begin{array}{c}\text { Textural } \\
\text { class }\end{array}$ & $\mathrm{pH}$ & $\begin{array}{c}\text { Exch. } \\
\text { Acidity c } \\
\text { mol }(\mathrm{P}+) \\
\mathrm{kg}-1\end{array}$ & $\begin{array}{c}\text { Exch. Al } \\
\text { c mol } \\
(\mathrm{P}+) \mathrm{kg}- \\
1\end{array}$ & $\begin{array}{c}\text { Exch. Ca } \\
\text { c mol (P+) } \\
\text { kg-1 }\end{array}$ & $\begin{array}{c}\text { Exch. } \\
\mathrm{Mg} \mathrm{c} \\
\mathrm{mol}(\mathrm{P}+) \\
\mathrm{kg}-1\end{array}$ & $\begin{array}{c}\text { CEC c } \\
\text { mol }(\mathrm{P}+) \\
\mathrm{kg}-1\end{array}$ & $\begin{array}{l}\text { OC } \\
(\%)\end{array}$ & $\begin{array}{c}\text { Av. N } \\
(\mathrm{kg} \mathrm{ha}-1)\end{array}$ & $\begin{array}{c}\text { Av.P } \\
(\mathrm{kg} \\
\text { ha-1) }\end{array}$ & $\begin{array}{c}\text { Av.K } \\
(\mathrm{kg} \\
\text { ha-1) }\end{array}$ & $\begin{array}{c}\text { LR } \\
(\mathrm{CaCO} \\
\text { 3) (q } \\
\text { ha-1) }\end{array}$ \\
\hline S1 & 81.4 & 7.0 & 11.6 & $\begin{array}{l}\text { Sandy } \\
\text { loam }\end{array}$ & 4.15 & 0.86 & 0.55 & 1.32 & 0.32 & 3.2 & 0.47 & 137.5 & 8.9 & 200.5 & 58.0 \\
\hline $\mathrm{S} 2$ & 74.6 & 7.8 & 17.6 & $\begin{array}{l}\text { Sandy } \\
\text { loam }\end{array}$ & 5.03 & 0.40 & 0.22 & 1.37 & 0.40 & 3.8 & 0.45 & 125.0 & 12.2 & 162.1 & 48.0 \\
\hline S3 & 75.8 & 4.1 & 20.1 & $\begin{array}{c}\text { Sandy clay } \\
\text { loam }\end{array}$ & 5.82 & 0.36 & 0 & 1.50 & 0.48 & 4.5 & 0.58 & 158.5 & 15.7 & 323.6 & 33.0 \\
\hline
\end{tabular}

Table.2 Chemical composition of Udaipur rock phosphate (URP) used in this study

\begin{tabular}{|l|l|}
\hline Parameter & Magnitude (\%) \\
\hline $\mathbf{P}$ & 7.8 \\
\hline $\mathbf{S}$ & 1.2 \\
\hline $\mathbf{C a}$ & 25.6 \\
\hline $\mathbf{M g}$ & 0.26 \\
\hline $\mathbf{K}$ & 0.24 \\
\hline
\end{tabular}

Table.3 Chemical composition of farmyard manure used in this study

\begin{tabular}{|c|c|}
\hline Parameter & Magnitude(\%) \\
\hline $\mathrm{N}$ & 1.2 \\
\hline O.C. & 0.75 \\
\hline $\mathrm{P}$ & 0.006 \\
\hline $\mathrm{K}$ & 0.25 \\
\hline $\mathrm{Ca}$ & 0.045 \\
\hline
\end{tabular}


Table.4 Change in soil $\mathrm{pH}$ at different cuttings

\begin{tabular}{|c|c|c|c|c|c|c|c|c|c|c|}
\hline \multirow[t]{2}{*}{ Soils } & \multirow[t]{2}{*}{ Treatments } & \multicolumn{9}{|c|}{ Soil pH } \\
\hline & & 1st & 2nd & 3rd & 4th & 5 th & 6th & 7th & 8th & Mean \\
\hline \multirow{6}{*}{$\begin{array}{l}\mathrm{S}_{1} \\
\text { (Initial } \\
\text { soil } \\
\text { pH-4.15) }\end{array}$} & $\mathrm{S}_{1} \mathrm{~T}_{1}=$ control $\mathrm{P}$ & 4.08 & 4.16 & 4.25 & 4.27 & 4.27 & 4.29 & 4.30 & 4.31 & 4.24 \\
\hline & $\mathrm{S}_{1} \mathrm{~T}_{2}=200 \% \mathrm{P}(\mathrm{URP})$ & 4.93 & 5.58 & 5.47 & 5.63 & 5.51 & 5.36 & 5.29 & 5.16 & 5.37 \\
\hline & $\mathrm{S}_{1} \mathrm{~T}_{3}=50 \% \mathrm{P}(\mathrm{URP})+50 \% \mathrm{P}(\mathrm{SSP})$ & 4.61 & 5.67 & 5.66 & 5.52 & 5.32 & 5.18 & 5.01 & 4.68 & 5.21 \\
\hline & $\mathrm{S}_{1} \mathrm{~T}_{4}=100 \% \mathrm{P}(\mathrm{URP})+\mathrm{OM}$ & 4.79 & 5.22 & 5.32 & 5.27 & 5.31 & 5.23 & 4.94 & 4.77 & 5.11 \\
\hline & $\mathrm{S}_{1} \mathrm{~T}_{5}=100 \% \mathrm{P}(\mathrm{URP})+\mathrm{Biof}$ & 4.68 & 5.13 & 5.29 & 5.22 & 5.25 & 5.16 & 4.91 & 4.72 & 5.05 \\
\hline & $\mathrm{S}_{1} \mathrm{~T}_{6}=100 \% \mathrm{P}(\mathrm{URP})+$ Lime & 5.12 & 5.76 & 5.91 & 5.85 & 5.67 & 5.43 & 5.26 & 4.92 & 5.49 \\
\hline \multirow{6}{*}{$\begin{array}{l}\mathbf{S}_{2} \\
\text { (Initial } \\
\text { soil } \\
\text { pH-5.03) }\end{array}$} & $\mathrm{S}_{2} \mathrm{~T}_{1}=$ control $\mathrm{P}$ & 4.95 & 5.19 & 5.11 & 5.17 & 5.12 & 5.09 & 5.17 & 5.16 & 5.12 \\
\hline & $\mathrm{S}_{2} \mathrm{~T}_{2}=200 \% \mathrm{P}(\mathrm{URP})$ & 5.87 & 6.33 & 6.35 & 6.41 & 6.34 & 6.25 & 6.04 & 5.88 & 6.18 \\
\hline & $\mathrm{S}_{2} \mathrm{~T}_{3}=50 \% \mathrm{P}(\mathrm{URP})+50 \% \mathrm{P}(\mathrm{SSP})$ & 5.56 & 6.44 & 6.59 & 6.42 & 6.21 & 6.02 & 5.81 & 5.59 & 6.08 \\
\hline & $\mathrm{S}_{2} \mathrm{~T}_{4}=100 \% \mathrm{P}(\mathrm{URP})+\mathrm{OM}$ & 5.96 & 6.08 & 6.14 & 5.81 & 5.62 & 5.49 & 5.37 & 5.62 & 5.76 \\
\hline & $\mathrm{S}_{2} \mathrm{~T}_{5}=100 \% \mathrm{P}(\mathrm{URP})+\mathrm{Biof}$ & 5.78 & 5.8 & 5.85 & 5.73 & 5.56 & 5.34 & 5.31 & 5.54 & 5.61 \\
\hline & $\mathrm{S}_{2} \mathrm{~T}_{6}=100 \% \mathrm{P}(\mathrm{URP})+$ Lime & 5.94 & 6.28 & 6.43 & 6.40 & 6.27 & 6.12 & 6.06 & 5.77 & 6.16 \\
\hline \multirow{6}{*}{$\begin{array}{l}\mathbf{S}_{3} \\
\text { (Initial } \\
\text { soil } \\
\text { pH-5.82) }\end{array}$} & $\mathrm{S}_{3} \mathrm{~T}_{1}=$ control P & 5.75 & 5.97 & 5.95 & 6.04 & 6.05 & 5.94 & 5.97 & 5.93 & 5.95 \\
\hline & $\mathrm{S}_{3} \mathrm{~T}_{2}=200 \% \mathrm{P}(\mathrm{URP})$ & 6.74 & 6.99 & 7.03 & 7.14 & 7.12 & 6.97 & 6.79 & 6.58 & 6.92 \\
\hline & $\mathrm{S}_{3} \mathrm{~T}_{3}=50 \% \mathrm{P}(\mathrm{URP})+50 \% \mathrm{P}(\mathrm{SSP})$ & 6.13 & 7.17 & 7.22 & 7.27 & 6.92 & 6.68 & 6.43 & 6.28 & 6.76 \\
\hline & $\mathrm{S}_{3} \mathrm{~T}_{4}=100 \% \mathrm{P}(\mathrm{URP})+\mathrm{OM}$ & 6.46 & 6.72 & 6.83 & 6.78 & 6.57 & 6.54 & 6.47 & 6.35 & 6.59 \\
\hline & $\mathrm{S}_{3} \mathrm{~T}_{5}=100 \% \mathrm{P}(\mathrm{URP})+\mathrm{Biof}$ & 6.37 & 6.53 & 6.66 & 6.63 & 6.51 & 6.48 & 6.44 & 6.29 & 6.49 \\
\hline & $\mathrm{S}_{3} \mathrm{~T}_{6}=100 \% \mathrm{P}(\mathrm{URP})+$ Lime & 6.87 & 7.18 & 7.29 & 7.15 & 6.89 & 6.95 & 6.87 & 6.44 & 6.96 \\
\hline $\mathrm{CD}(0.05)$ & $\begin{array}{l}\mathrm{S} \\
\mathrm{T} \\
\mathrm{SXT}\end{array}$ & $\begin{array}{l}0.09 \\
0.13 \\
\text { NS }\end{array}$ & $\begin{array}{l}0.15 \\
0.21 \\
\text { NS }\end{array}$ & $\begin{array}{l}0.23 \\
0.32 \\
\text { NS }\end{array}$ & $\begin{array}{l}0.18 \\
0.25 \\
\text { NS }\end{array}$ & $\begin{array}{l}0.15 \\
0.21 \\
\text { NS }\end{array}$ & $\begin{array}{l}0.15 \\
0.21 \\
\text { NS }\end{array}$ & $\begin{array}{l}0.13 \\
0.18 \\
\text { NS }\end{array}$ & $\begin{array}{l}0.15 \\
0.21 \\
\text { NS }\end{array}$ & $\begin{array}{l}- \\
- \\
-\end{array}$ \\
\hline C.V. $(\%)$ & & 1.99 & 2.89 & 4.40 & 3.47 & 2.87 & 3.03 & 2.63 & 3.07 & - \\
\hline
\end{tabular}


Table.5 Change in soil available phosphorus $\left(\mathrm{kg} \mathrm{ha}^{-1}\right)$ at different cuttings

\begin{tabular}{|c|c|c|c|c|c|c|c|c|c|c|c|}
\hline \multirow[t]{2}{*}{ Soils } & \multirow[t]{2}{*}{ Treatments } & \multicolumn{10}{|c|}{ Available $\left(\mathrm{kg} \mathrm{ha}^{-1}\right)$} \\
\hline & & 1st & 2nd & 3rd & 4th & 5 th & 6th & 7th & 8th & Mean & $\begin{array}{c}\text { P build } \\
\text { up } \\
\left(\mathrm{kg} \mathrm{ha}^{-1}\right)\end{array}$ \\
\hline \multirow{6}{*}{$\begin{array}{c}\mathrm{S}_{1}(\mathrm{pH}=4.15) \\
\text { (Initial } \\
\text { Av.P=8.92 } \\
\left.\mathrm{kg} \mathrm{ha}^{-1}\right)\end{array}$} & $\mathrm{S}_{1} \mathrm{~T}_{1}=$ control $\mathrm{P}$ & 8.49 & 7.92 & 7.47 & 7.14 & 7.08 & 6.86 & 6.57 & 6.21 & 7.22 & -2.7 \\
\hline & $\mathrm{S}_{1} \mathrm{~T}_{2}=200 \% \mathrm{P}(\mathrm{URP})$ & 12.11 & 21.54 & 20.24 & 21.93 & 23.49 & 22.21 & 20.89 & 19.97 & 20.30 & 11.0 \\
\hline & $\begin{array}{c}\mathrm{S}_{1} \mathrm{~T}_{3}=50 \% \mathrm{P}(\mathrm{URP}) \\
+50 \% \mathrm{P}(\mathrm{SSP})\end{array}$ & 13.74 & 25.79 & 22.63 & 20.81 & 23.87 & 22.65 & 20.18 & 17.81 & 20.94 & 8.9 \\
\hline & $\begin{array}{c}\mathrm{S}_{1} \mathrm{~T}_{4}=100 \% \mathrm{P}(\mathrm{URP}) \\
+\mathrm{OM}\end{array}$ & 11.53 & 19.68 & 17.33 & 16.27 & 17.48 & 17.92 & 15.39 & 14.66 & 16.28 & 5.7 \\
\hline & $\begin{array}{c}\mathrm{S}_{1} \mathrm{~T}_{5}=100 \% \mathrm{P}(\mathrm{URP}) \\
+ \text { Biof }\end{array}$ & 11.26 & 19.23 & 16.98 & 16.03 & 17.34 & 17.24 & 14.56 & 13.72 & 15.80 & 4.8 \\
\hline & $\begin{aligned} \mathrm{S}_{1} \mathrm{~T}_{6}= & 100 \% \mathrm{P}(\mathrm{URP}) \\
& + \text { Lime }\end{aligned}$ & 13.85 & 24.87 & 23.79 & 19.86 & 21.84 & 19.59 & 16.05 & 15.75 & 19.45 & 6.83 \\
\hline \multirow{6}{*}{$\begin{array}{l}\mathrm{S}_{2}(\mathbf{p H}=5.03) \\
\text { Initial } \\
\text { Av.P=12.17 } \\
\left.\mathrm{kg} \mathrm{ha}^{-1}\right)\end{array}$} & $\mathrm{S}_{2} \mathrm{~T}_{1}=$ control $\mathrm{P}$ & 11.71 & 10.76 & 10.23 & 9.53 & 9.21 & 8.77 & 8.61 & 8.34 & 9.65 & -3.8 \\
\hline & $\mathrm{S}_{2} \mathrm{~T}_{2}=200 \% \mathrm{P}(\mathrm{URP})$ & 15.85 & 27.72 & 25.91 & 28.82 & 32.29 & 30.29 & 27.77 & 26.69 & 26.92 & 14.5 \\
\hline & $\begin{array}{c}\mathrm{S}_{2} \mathrm{~T}_{3}=50 \% \mathrm{P}(\mathrm{URP})+ \\
50 \% \mathrm{P}(\mathrm{SSP})\end{array}$ & 17.94 & 32.89 & 29.04 & 27.55 & 30.69 & 29.13 & 25.3 & 24.48 & 27.13 & 12.3 \\
\hline & $\begin{array}{c}\mathrm{S}_{2} \mathrm{~T}_{4}=100 \% \mathrm{P}(\mathrm{URP}) \\
+\mathrm{OM}\end{array}$ & 14.63 & 25.37 & 22.28 & 21.5 & 23.59 & 23.7 & 20.36 & 19.76 & 21.40 & 7.6 \\
\hline & $\begin{aligned} \mathrm{S}_{2} \mathrm{~T}_{5}= & 100 \% \mathrm{P}(\mathrm{URP}) \\
& + \text { Biof }\end{aligned}$ & 14.28 & 24.94 & 20.51 & 21.49 & 25.43 & 25.07 & 20.37 & 18.55 & 21.33 & 6.4 \\
\hline & $\begin{aligned} \mathrm{S}_{2} \mathrm{~T}_{6}= & 100 \% \mathrm{P}(\mathrm{URP}) \\
& + \text { Lime }\end{aligned}$ & 18.1 & 33.71 & 32.14 & 26.11 & 30.01 & 27.9 & 21.33 & 21.16 & 26.31 & 9.0 \\
\hline \multirow{6}{*}{$\begin{array}{c}\mathrm{S}_{3}(\mathrm{pH}=5.82) \\
\text { (Initial } \\
\text { Av. } \mathrm{P}=15.74 \\
\left.\mathrm{~kg} \mathrm{ha}^{-1}\right)\end{array}$} & $\mathrm{S}_{3} \mathrm{~T}_{1}=$ control $\mathrm{P}$ & 14.63 & 13.42 & 12.71 & 12.45 & 11.89 & 11.47 & 10.83 & 10.23 & 12.20 & -5.5 \\
\hline & $\mathrm{S}_{3} \mathrm{~T}_{2}=200 \% \mathrm{P}(\mathrm{URP})$ & 19.18 & 31.37 & 29.55 & 31.48 & 35.12 & 33.65 & 30.11 & 28.54 & 29.88 & 12.8 \\
\hline & $\begin{array}{c}\mathrm{S}_{3} \mathrm{~T}_{3}=50 \% \mathrm{P}(\mathrm{URP})+ \\
50 \% \mathrm{P}(\mathrm{SSP})\end{array}$ & 23.32 & 40.63 & 36.16 & 32.54 & 33.64 & 32.26 & 28.7 & 27.18 & 31.80 & 11.4 \\
\hline & $\begin{array}{c}\mathrm{S}_{3} \mathrm{~T}_{4}=100 \% \mathrm{P}(\mathrm{URP}) \\
+\mathrm{OM}\end{array}$ & 18.94 & 30.67 & 28.25 & 27.52 & 29.21 & 28.41 & 25.13 & 22.49 & 26.33 & 6.8 \\
\hline & $\begin{array}{c}\mathrm{S}_{3} \mathrm{~T}_{5}=100 \% \mathrm{P}(\mathrm{URP}) \\
+ \text { Biof }\end{array}$ & 18.43 & 29.89 & 28.49 & 26.51 & 27.31 & 26.75 & 22.85 & 20.38 & 25.08 & 4.6 \\
\hline & $\begin{aligned} \mathrm{S}_{3} \mathrm{~T}_{6}= & 100 \% \mathrm{P}(\mathrm{URP}) \\
& + \text { Lime }\end{aligned}$ & 21.67 & 33.52 & 31.34 & 28.79 & 30.74 & 30.41 & 27.36 & 23.87 & 28.46 & 8.1 \\
\hline \multirow[t]{2}{*}{$\mathrm{CD}(0.05)$} & $\mathrm{S}$ & 0.91 & 0.87 & 1.01 & 0.64 & 0.93 & 0.81 & 0.72 & 0.52 & 0.55 & - \\
\hline & $\begin{array}{c}\mathrm{T} \\
\text { SxT }\end{array}$ & $\begin{array}{l}1.28 \\
\mathrm{NS}\end{array}$ & $\begin{array}{l}1.23 \\
2.13\end{array}$ & $\begin{array}{l}1.43 \\
2.49\end{array}$ & $\begin{array}{l}0.91 \\
1.57\end{array}$ & $\begin{array}{l}1.31 \\
2.27\end{array}$ & $\begin{array}{l}1.15 \\
1.99\end{array}$ & $\begin{array}{l}1.02 \\
1.77\end{array}$ & $\begin{array}{l}0.73 \\
1.27\end{array}$ & 0.78 & - \\
\hline C.V.(\%) & - & 6.82 & 4.02 & 5.13 & 3.41 & 4.52 & 4.12 & 4.23 & 3.20 & 3.02 & - \\
\hline
\end{tabular}


Table.6 Change in exchangeable calcium $\left(\mathrm{cmol}(\mathrm{p})^{+} \mathrm{kg}^{-1}\right)$ of of soil at different cuttings

\begin{tabular}{|c|c|c|c|c|c|c|c|c|c|c|}
\hline \multirow[t]{2}{*}{ Soils } & \multirow[t]{2}{*}{ Treatments } & \multicolumn{9}{|c|}{ Exchangeable calcium $\left(\mathrm{cmol}(\mathrm{p})^{+} \mathrm{kg}^{-1}\right)$} \\
\hline & & $1 \mathrm{st}$ & 2 nd & $3 r d$ & 4th & 5 th & 6th & 7th & 8th & Mean \\
\hline \multirow{6}{*}{$\begin{array}{l}\mathrm{S}_{1}(\mathrm{pH}=4.15) \\
(\text { Initial } \\
\text { Ex.Ca=1.32) }\end{array}$} & $\mathrm{S}_{1} \mathrm{~T}_{1}=$ control P & 1.28 & 1.06 & 0.95 & 0.87 & 0.75 & 0.68 & 0.64 & 0.56 & 0.85 \\
\hline & $\mathrm{S}_{1} \mathrm{~T}_{2}=200 \% \mathrm{P}(\mathrm{URP})$ & 1.53 & 1.37 & 1.39 & 1.38 & 1.41 & 1.55 & 1.57 & 1.46 & 1.46 \\
\hline & $\mathrm{S}_{1} \mathrm{~T}_{3}=50 \% \mathrm{P}(\mathrm{URP})+50 \% \mathrm{P}(\mathrm{SSP})$ & 1.89 & 1.54 & 1.48 & 1.41 & 1.52 & 1.57 & 1.46 & 1.42 & 1.54 \\
\hline & $\mathrm{S}_{1} \mathrm{~T}_{4}=100 \% \mathrm{P}(\mathrm{URP})+\mathrm{OM}$ & 1.46 & 1.19 & 1.26 & 1.17 & 1.28 & 1.30 & 1.27 & 1.22 & 1.27 \\
\hline & $\mathrm{S}_{1} \mathrm{~T}_{5}=100 \% \mathrm{P}(\mathrm{URP})+\mathrm{Biof}$ & 1.38 & 1.11 & 1.21 & 1.09 & 1.19 & 1.19 & 1.23 & 1.15 & 1.19 \\
\hline & $\mathrm{S}_{1} \mathrm{~T}_{6}=100 \% \mathrm{P}(\mathrm{URP})+$ Lime & 1.67 & 1.59 & 1.53 & 1.47 & 1.41 & 1.39 & 1.40 & 1.34 & 1.48 \\
\hline \multirow{6}{*}{$\begin{array}{l}\mathrm{S}_{2}(\mathrm{pH}=5.03) \\
(\text { Initial } \\
\text { Ex.Ca=1.37) }\end{array}$} & $\mathrm{S}_{2} \mathrm{~T}_{1}=$ control $\mathrm{P}$ & 1.31 & 1.09 & 0.94 & 0.85 & 0.88 & 0.77 & 0.75 & 0.61 & 0.90 \\
\hline & $\mathrm{S}_{2} \mathrm{~T}_{2}=200 \% \mathrm{P}(\mathrm{URP})$ & 1.61 & 1.46 & 1.43 & 1.40 & 1.48 & 1.61 & 1.64 & 1.55 & 1.52 \\
\hline & $\mathrm{S}_{2} \mathrm{~T}_{3}=50 \% \mathrm{P}(\mathrm{URP})+50 \% \mathrm{P}(\mathrm{SSP})$ & 2.02 & 1.61 & 1.51 & 1.44 & 1.63 & 1.66 & 1.52 & 1.51 & 1.61 \\
\hline & $\mathrm{S}_{2} \mathrm{~T}_{4}=100 \% \mathrm{P}(\mathrm{URP})+\mathrm{OM}$ & 1.53 & 1.22 & 1.32 & 1.12 & 1.23 & 1.34 & 1.28 & 1.24 & 1.29 \\
\hline & $\mathrm{S}_{2} \mathrm{~T}_{5}=100 \% \mathrm{P}(\mathrm{URP})+\mathrm{Biof}$ & 1.44 & 1.16 & 1.23 & 1.07 & 1.17 & 1.25 & 1.19 & 1.18 & 1.21 \\
\hline & $\mathrm{S}_{2} \mathrm{~T}_{6}=100 \% \mathrm{P}(\mathrm{URP})+$ Lime & 1.75 & 1.64 & 1.57 & 1.51 & 1.44 & 1.47 & 1.43 & 1.45 & 1.53 \\
\hline \multirow{6}{*}{$\begin{array}{l}\mathrm{S}_{3}(\mathrm{pH}=5.82) \\
(\text { Initial } \\
\text { Ex.Ca=1.50) }\end{array}$} & $\mathrm{S}_{3} \mathrm{~T}_{1}=$ control P & 1.42 & 1.27 & 1.16 & 1.06 & 0.91 & 0.89 & 0.87 & 0.71 & 1.04 \\
\hline & $\mathrm{S}_{3} \mathrm{~T}_{2}=200 \% \mathrm{P}(\mathrm{URP})$ & 1.70 & 1.39 & 1.23 & 1.38 & 1.43 & 1.56 & 1.68 & 1.63 & 1.50 \\
\hline & $\mathrm{S}_{3} \mathrm{~T}_{3}=50 \% \mathrm{P}(\mathrm{URP})+50 \% \mathrm{P}(\mathrm{SSP})$ & 2.15 & 1.67 & 1.59 & 1.51 & 1.71 & 1.78 & 1.86 & 1.75 & 1.75 \\
\hline & $\mathrm{S}_{3} \mathrm{~T}_{4}=100 \% \mathrm{P}(\mathrm{URP})+\mathrm{OM}$ & 1.61 & 1.30 & 1.35 & 1.17 & 1.26 & 1.31 & 1.33 & 1.37 & 1.34 \\
\hline & $\mathrm{S}_{3} \mathrm{~T}_{5}=100 \% \mathrm{P}(\mathrm{URP})+\mathrm{Biof}$ & 1.55 & 1.27 & 1.29 & 1.10 & 1.20 & 1.23 & 1.21 & 1.26 & 1.26 \\
\hline & $\mathrm{S}_{3} \mathrm{~T}_{6}=100 \% \mathrm{P}(\mathrm{URP})+$ Lime & 1.85 & 1.76 & 1.69 & 1.61 & 1.55 & 1.52 & 1.55 & 1.50 & 1.63 \\
\hline C.D.(0.05) & $\begin{array}{l}\mathrm{S} \\
\mathrm{T} \\
\text { SxT }\end{array}$ & $\begin{array}{l}0.09 \\
0.12 \\
\text { NS }\end{array}$ & $\begin{array}{l}0.08 \\
0.12 \\
\text { NS }\end{array}$ & $\begin{array}{c}\text { NS } \\
0.11 \\
\text { NS }\end{array}$ & $\begin{array}{l}\text { NS } \\
0.10 \\
\text { NS }\end{array}$ & $\begin{array}{l}\text { NS } \\
0.10 \\
\text { NS }\end{array}$ & $\begin{array}{c}\text { NS } \\
0.13 \\
\text { NS }\end{array}$ & $\begin{array}{c}0.08 \\
0.11 \\
\text { NS }\end{array}$ & $\begin{array}{c}0.09 \\
0.12 \\
\text { NS }\end{array}$ & $\begin{array}{l}0.03 \\
0.04 \\
0.07\end{array}$ \\
\hline C.V.(\%) & - & 6.16 & 7.25 & 6.69 & 6.87 & 6.58 & 8.30 & 6.91 & 8.00 & 2.37 \\
\hline
\end{tabular}


Table.7 Dry weight of hybrid napier grass $\left(\mathrm{g} \mathrm{pot}^{-1}\right)$ at different cuttings

\begin{tabular}{|c|c|c|c|c|c|c|c|c|c|c|c|c|}
\hline \multirow[t]{2}{*}{ Soils } & \multirow[t]{2}{*}{ Treatments } & \multicolumn{11}{|c|}{ Dry matter yield $\left(\mathrm{g} \mathrm{pot}^{-1}\right)$} \\
\hline & & 1st & 2nd & 3rd & 4th & 5 th & 6th & 7 th & 8th & Total & $\begin{array}{c}\% \\
\text { increase } \\
\text { in yield } \\
\text { over } \\
\text { control }\end{array}$ & $\begin{array}{c}\text { RAE } \\
(\%)\end{array}$ \\
\hline \multirow{6}{*}{$\begin{array}{l}S_{1}(\mathrm{pH}=4 . \\
15)\end{array}$} & $\mathrm{S}_{1} \mathrm{~T}_{1}=$ control $\mathrm{P}$ & 8.44 & 7.81 & 5.93 & 5.78 & 6.53 & 6.10 & 4.83 & 3.18 & $48.54^{\mathrm{a}}$ & - & - \\
\hline & $\mathrm{S}_{1} \mathrm{~T}_{2}=200 \% \mathrm{P}(\mathrm{URP})$ & 11.27 & 9.49 & 8.12 & 8.09 & 10.40 & 9.31 & 7.47 & 5.67 & $69.80^{\text {fgh }}$ & 43.7 & 107 \\
\hline & $\begin{array}{l}\mathrm{S}_{1} \mathrm{~T}_{3}=50 \% \mathrm{P}(\mathrm{URP})+50 \% \mathrm{P}( \\
\mathrm{SSP})\end{array}$ & 11.55 & 10.05 & 8.85 & 7.27 & 10.01 & 9.03 & 6.64 & 4.97 & $68.35^{\mathrm{fg}}$ & 40.7 & 100 \\
\hline & $\mathrm{S}_{1} \mathrm{~T}_{4}=100 \% \mathrm{P}(\mathrm{URP})+\mathrm{OM}$ & 10.93 & 9.04 & 7.61 & 6.92 & 9.17 & 8.21 & 6.11 & 4.42 & $62.38^{\text {cde }}$ & 28.4 & 70 \\
\hline & $\mathrm{S}_{1} \mathrm{~T}_{5}=100 \% \mathrm{P}(\mathrm{URP})+\mathrm{Biof}$ & 10.63 & 8.63 & 7.46 & 6.56 & 8.61 & 7.80 & 5.82 & 4.33 & $59.82^{\text {cd }}$ & 23.2 & 57 \\
\hline & $\mathrm{S}_{1} \mathrm{~T}_{6}=100 \% \mathrm{P}(\mathrm{URP})+$ Lime & 12.55 & 11.95 & 8.15 & 6.84 & 9.87 & 8.57 & 6.34 & 4.66 & $68.89^{\mathrm{fgh}}$ & 41.8 & 103 \\
\hline \multirow{6}{*}{$\begin{array}{l}\mathrm{S}_{2}(\mathrm{pH}=5 . \\
\mathbf{0 3})\end{array}$} & $\mathrm{S}_{2} \mathrm{~T}_{1}=$ control P & 9.94 & 8.61 & 6.35 & 6.52 & 6.76 & 6.94 & 5.27 & 3.37 & $53.73^{\mathrm{b}}$ & - & 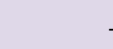 \\
\hline & $\mathrm{S}_{2} \mathrm{~T}_{2}=200 \% \mathrm{P}(\mathrm{URP})$ & 12.61 & 10.35 & 9.45 & 8.94 & 10.11 & 9.70 & 8.17 & 6.30 & $75.61^{\mathrm{ij}}$ & 40.7 & 108 \\
\hline & $\begin{array}{l}\mathrm{S}_{2} \mathrm{~T}_{3}=50 \% \mathrm{P}(\mathrm{URP})+50 \% \mathrm{P}( \\
\mathrm{SSP})\end{array}$ & 13.41 & 10.80 & 10.21 & 7.89 & 9.62 & 9.54 & 7.27 & 5.31 & $74.02^{\text {hij }}$ & 37.7 & 100 \\
\hline & $\mathrm{S}_{2} \mathrm{~T}_{4}=100 \% \mathrm{P}(\mathrm{URP})+\mathrm{OM}$ & 12.56 & 9.76 & 8.35 & 7.54 & 9.13 & 8.69 & 6.46 & 4.74 & $67.21^{\mathrm{ef}}$ & 25.1 & 60 \\
\hline & $\mathrm{S}_{2} \mathrm{~T}_{5}=100 \% \mathrm{P}(\mathrm{URP})+\mathrm{Biof}$ & 12.20 & 9.44 & 8.23 & 7.34 & 8.72 & 8.23 & 6.20 & 4.37 & $64.71^{\text {def }}$ & 20.4 & 54 \\
\hline & $\mathrm{S}_{2} \mathrm{~T}_{6}=100 \% \mathrm{P}(\mathrm{URP})+$ Lime & 13.89 & 12.70 & 9.61 & 7.47 & 9.45 & 9.01 & 6.71 & 4.95 & $73.77^{\text {ghij }}$ & 37.3 & 99 \\
\hline \multirow{6}{*}{$\begin{array}{l}S_{3}(\mathrm{pH}=5 . \\
82)\end{array}$} & $\mathrm{S}_{3} \mathrm{~T}_{1}=$ control $\mathrm{P}$ & 10.22 & 9.14 & 8.08 & 6.61 & 7.27 & 7.09 & 5.80 & 3.48 & $57.68^{\mathrm{bc}}$ & - & 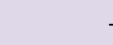 \\
\hline & $\mathrm{S}_{3} \mathrm{~T}_{2}=200 \% \mathrm{P}(\mathrm{URP})$ & 12.78 & 10.85 & 11.24 & 8.88 & 9.49 & 9.80 & 8.57 & 6.65 & $78.24^{\mathrm{ij}}$ & 35.7 & 76 \\
\hline & $\begin{array}{l}\mathrm{S}_{3} \mathrm{~T}_{3}=50 \% \mathrm{P}(\mathrm{URP})+50 \% \mathrm{P}( \\
\mathrm{SSP})\end{array}$ & 14.58 & 13.39 & 12.95 & 8.13 & 10.17 & $\begin{array}{r}10.3 \\
2\end{array}$ & 9.04 & 6.08 & $84.64^{\mathrm{k}}$ & 46.7 & 100 \\
\hline & $\mathrm{S}_{3} \mathrm{~T}_{4}=100 \% \mathrm{P}(\mathrm{URP})+\mathrm{OM}$ & 13.22 & 11.35 & 11.70 & 7.70 & 9.08 & 9.01 & 8.21 & 5.11 & $75.36^{\mathrm{ij}}$ & 30.6 & 66 \\
\hline & $\mathrm{S}_{3} \mathrm{~T}_{5}=100 \% \mathrm{P}(\mathrm{URP})+\mathrm{Biof}$ & 12.86 & 11.11 & 12.20 & 7.24 & 8.83 & 8.50 & 8.09 & 4.51 & $73.33^{\text {ghi }}$ & 27.1 & 58 \\
\hline & $\mathrm{S}_{3} \mathrm{~T}_{6}=100 \% \mathrm{P}(\mathrm{URP})+$ Lime & 14.16 & 13.07 & 11.85 & 7.52 & 9.14 & 9.17 & 8.44 & 5.81 & $79.14^{\mathrm{j}}$ & 37.2 & 80 \\
\hline \multirow[t]{3}{*}{$\mathrm{CD}(0.05)$} & $S$ & 0.58 & 0.64 & 0.55 & 0.51 & NS & 0.53 & 0.59 & 0.36 & 2.04 & - & - \\
\hline & $\mathrm{T}$ & 0.27 & 0.90 & 0.78 & 0.73 & 1.09 & 0.76 & 0.83 & 0.51 & 2.89 & & \\
\hline & SXT & NS & NS & NS & NS & NS & NS & NS & NS & NS & & \\
\hline $\operatorname{CV}(\%)$ & & 5.54 & 7.08 & 6.99 & 8.05 & 9.97 & 7.19 & 9.74 & 8.57 & 3.47 & - & - \\
\hline
\end{tabular}


Fig.1 Change in soil $\mathrm{pH}$ at different cuttings

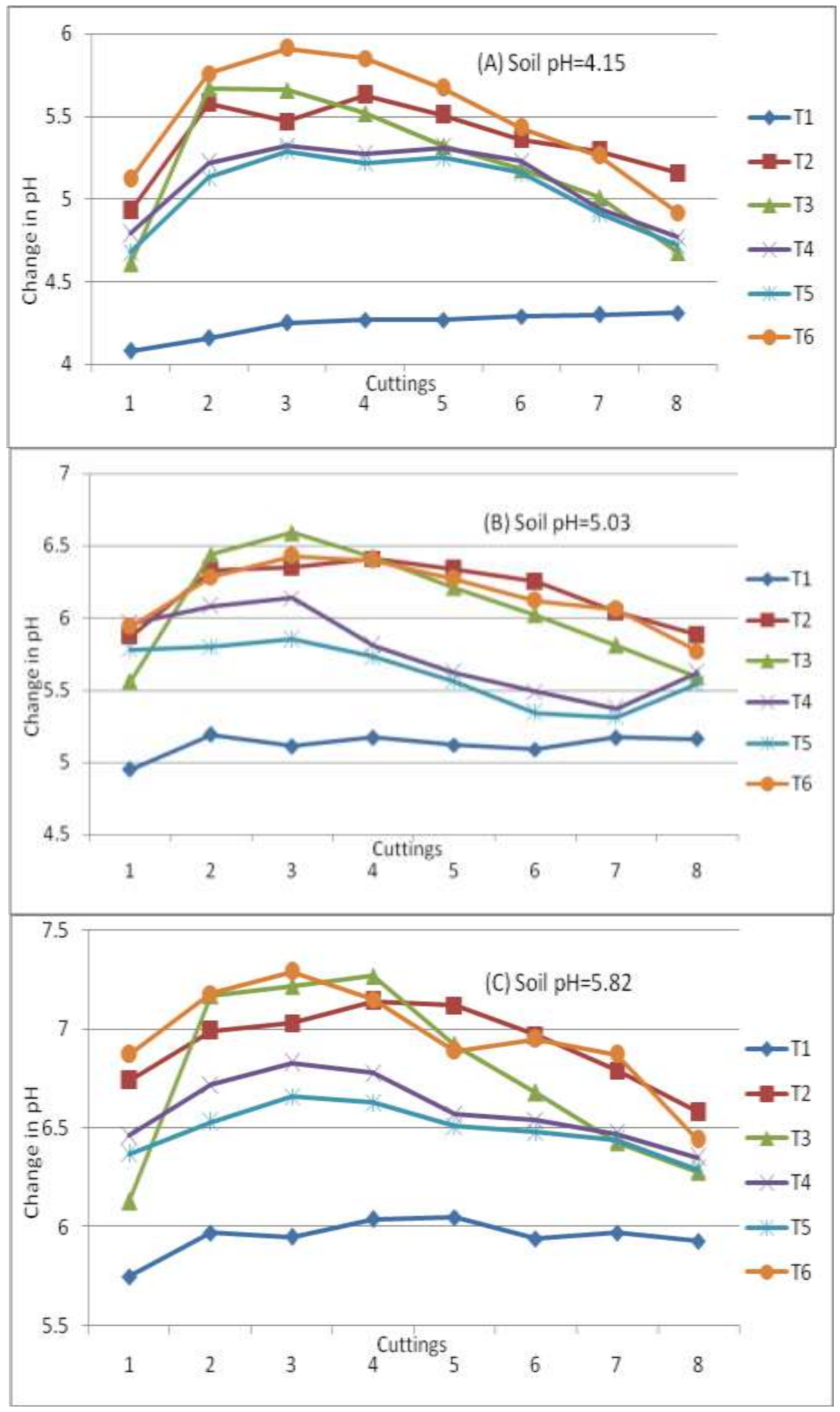


Fig.2 Change in available phosphorus of soil at different cuttings

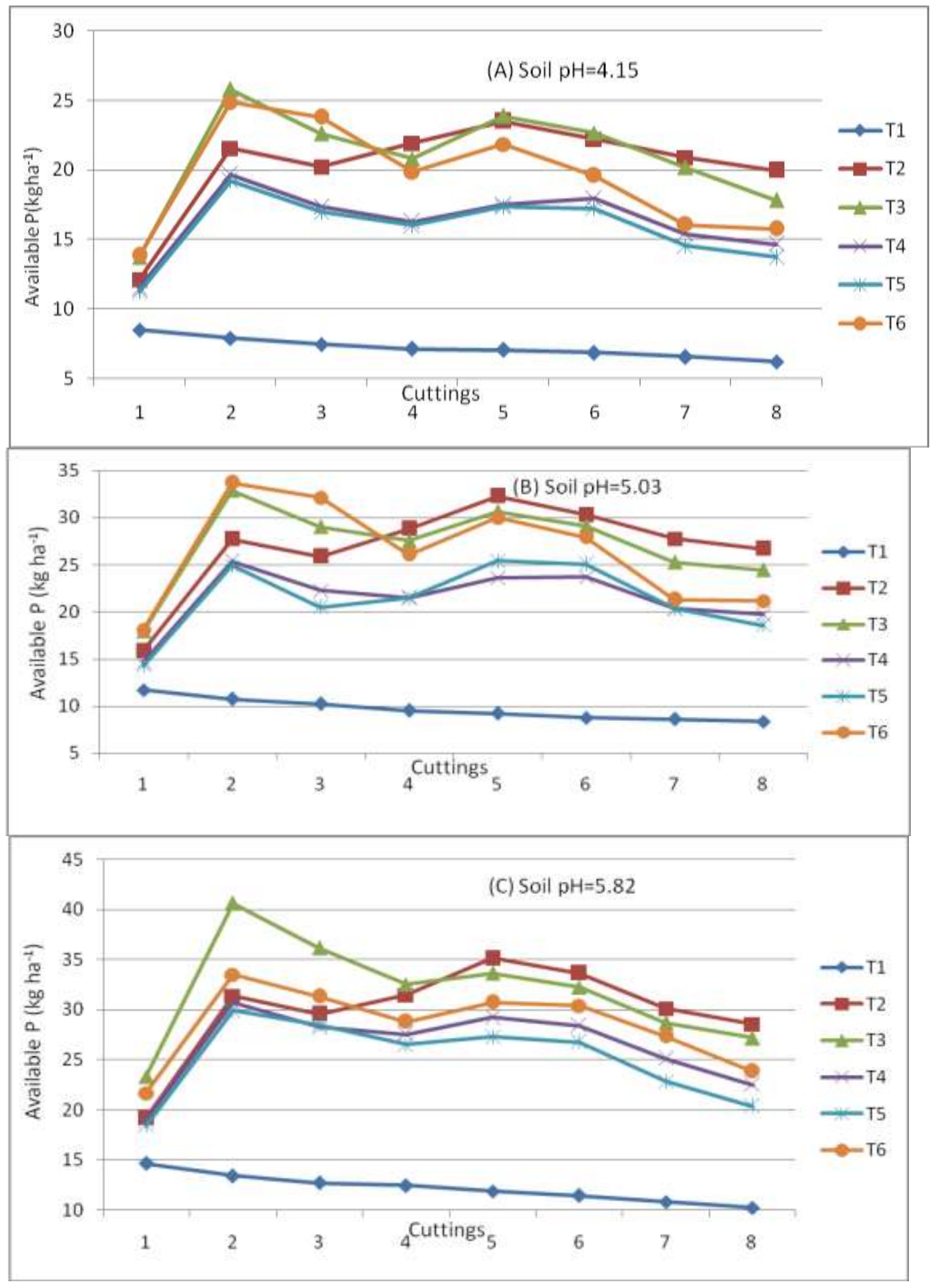


Fig.3 Change in exchangeable calcium of soil at different cuttings

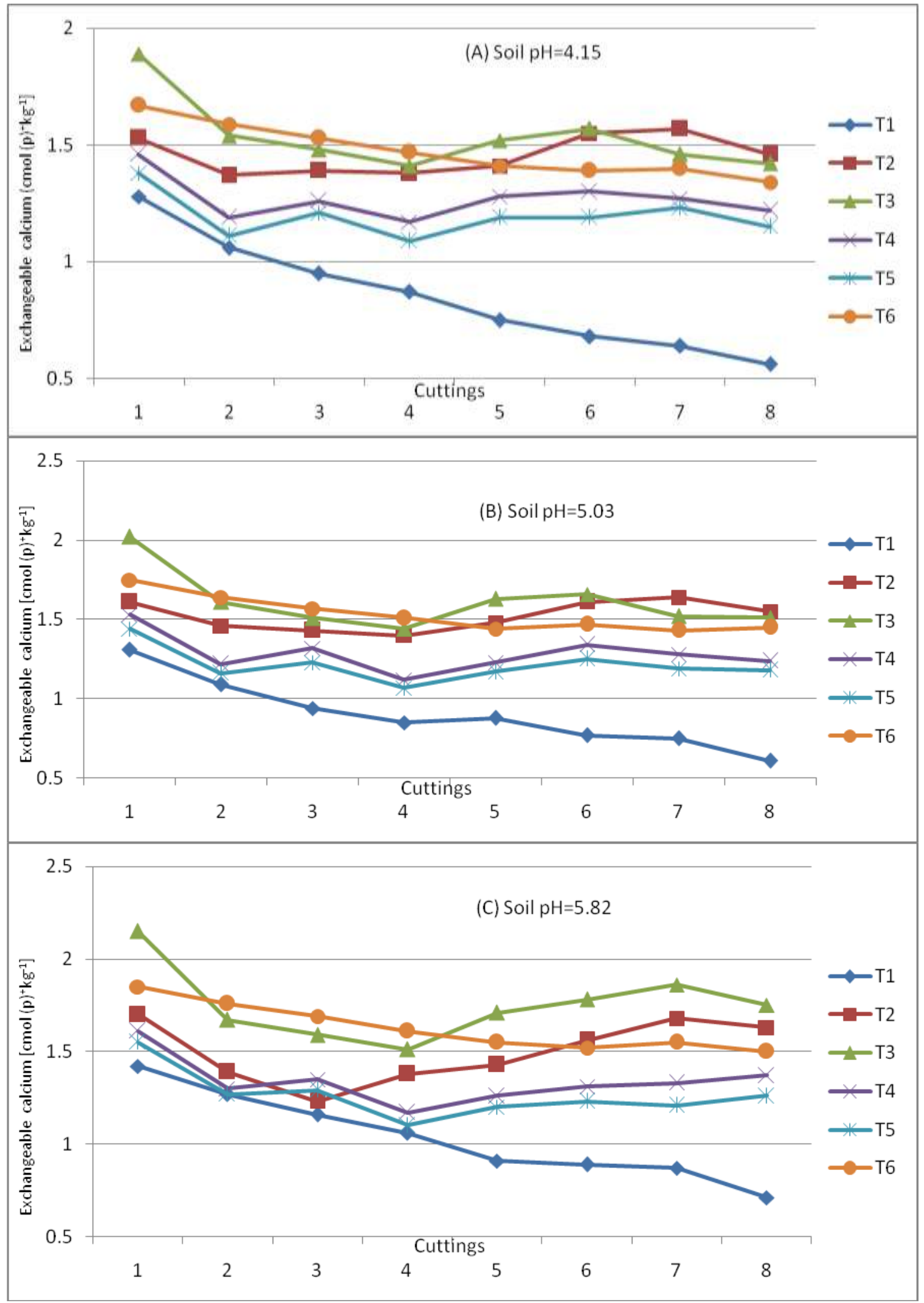


Fig.4 Effect of treatments on cumulative biomass yield of hybrid napier grass $\left(\mathrm{g} \mathrm{pot}^{-1}\right.$

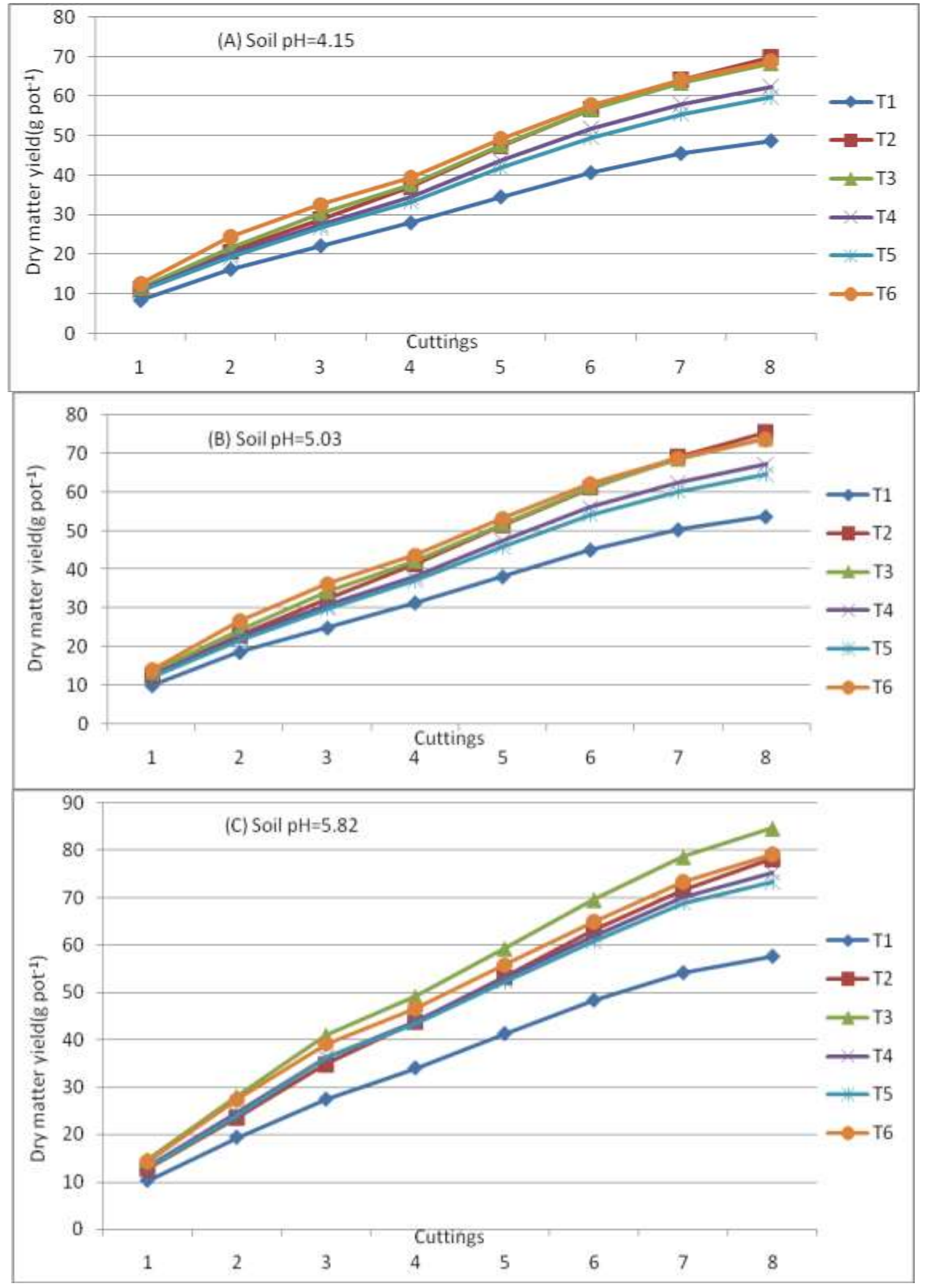


Fig.5 Total biomass yield of hybrid napier grass $\left(\mathrm{g} \mathrm{pot}^{-1}\right)$ as influenced by soil $\mathrm{pH}$

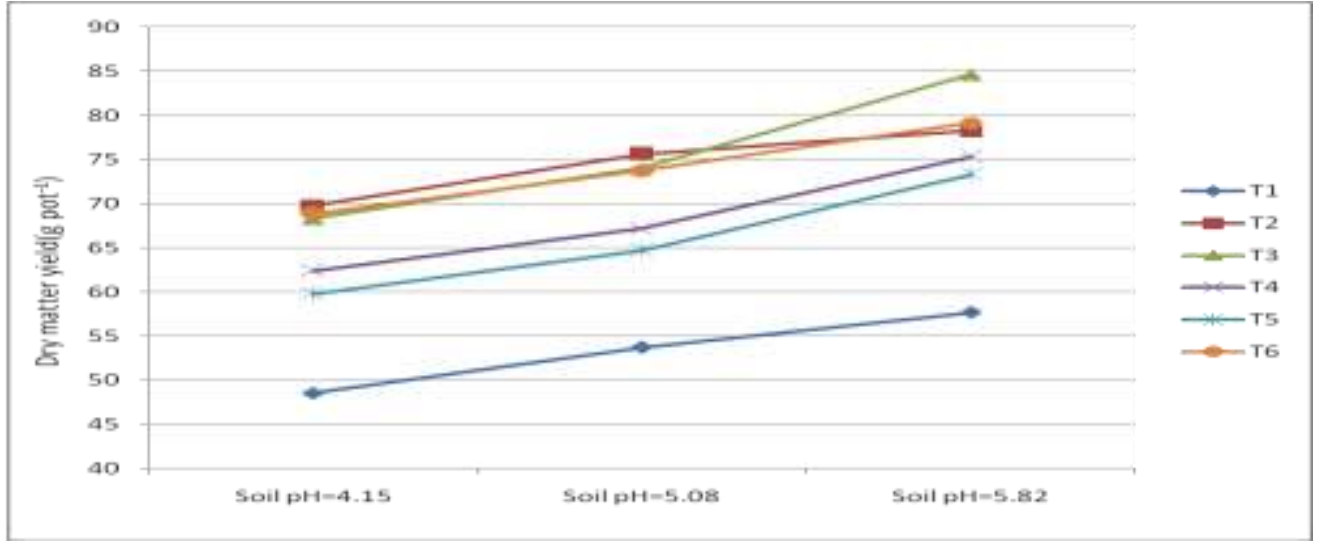

Fig.6 Correlations between mean soil available $\mathrm{P}$ and total dry matter yield of hybrid napier grass

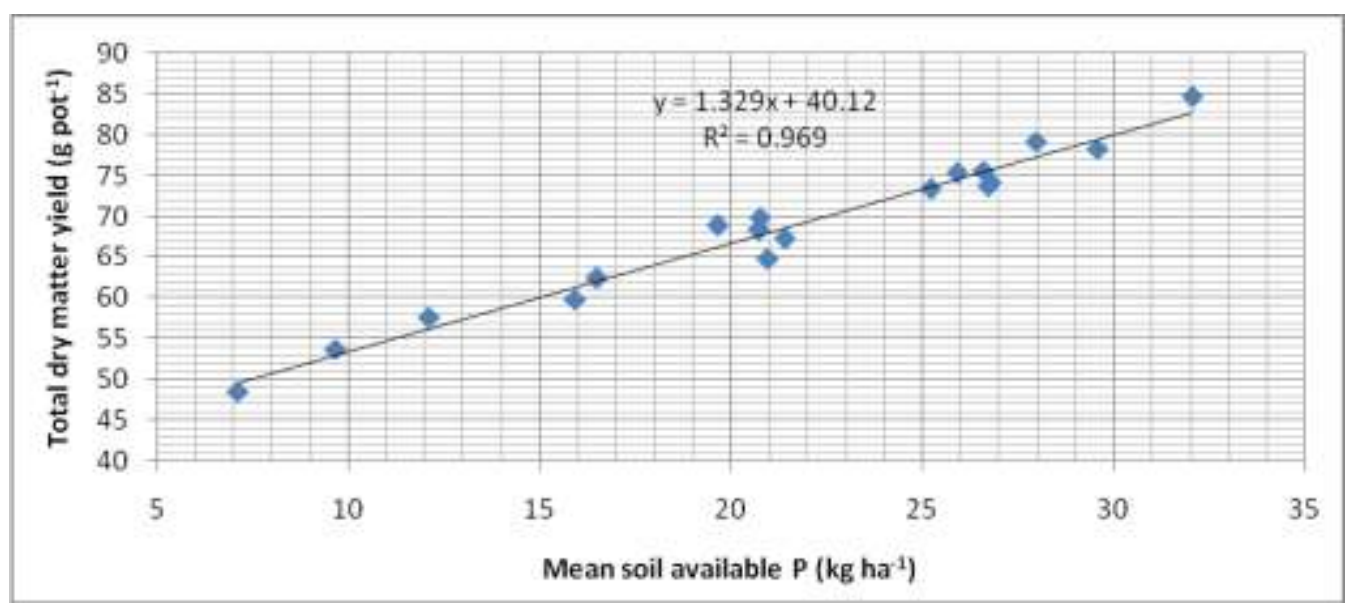

Fig.7 Correlations between mean soil exchangeable $\mathrm{Ca}$ and total dry matter yield of hybrid napier grass

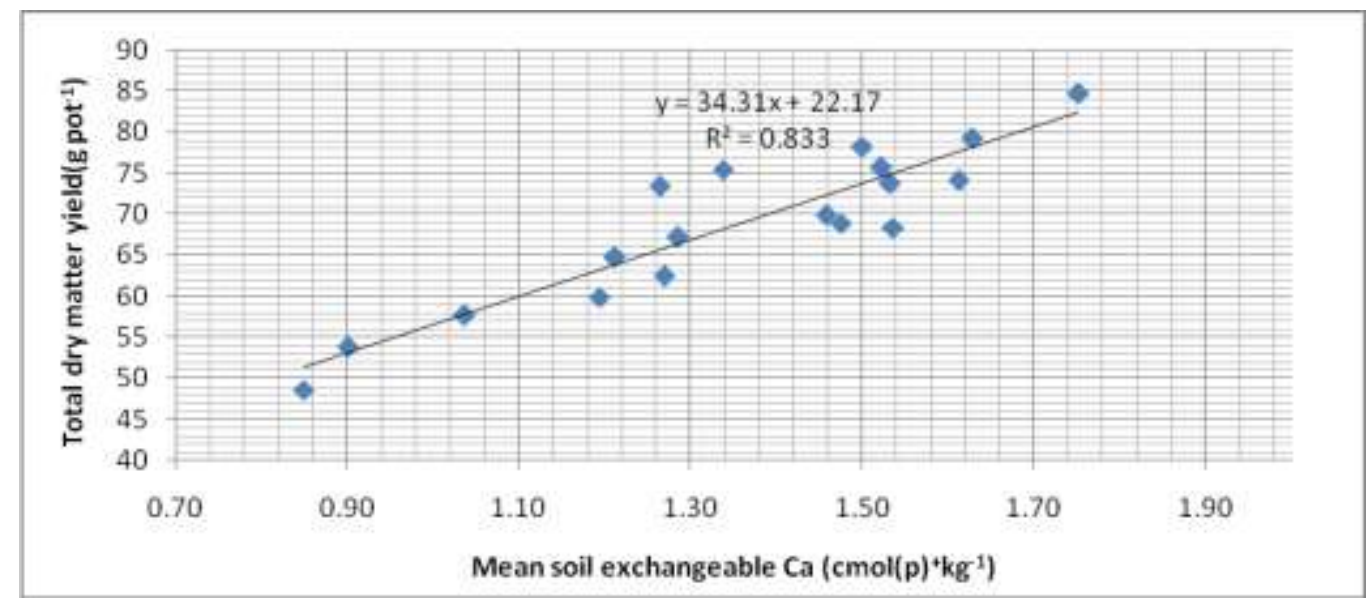


At the end of $8^{\text {th }}$ cutting, exchangeable calcium content in control decreased by $52-58$ $\%$ over the initial value might be due to crop removal from the native soil source. Sole application of higher dose of URP $\left(\mathrm{T}_{2}\right)$ increased calcium content significantly over all other treatments but was at par with $T_{3}$ (URP+ SSP) treatment at the end of $8^{\text {th }}$ cutting. Addition of lime with URP recorded higher calcium content in soil than URP+ FYM or URP+PSB but was at par with URP+ SSP treatment (Table 6 and Fig. 3).

\section{Biomass yield of hybrid napier grass at different stages of cutting}

The cumulative biomass yield of hybrid Napier grass increased significantly in URP treatments compared to that in the control. The magnitude of increase varied from 23-44 $\%$ in $\mathrm{S}_{1}, 20-41 \%$ in $\mathrm{S}_{2}$, and $27-47 \%$ in $\mathrm{S}_{3}$ (Table 7 and Fig. 4).

Sole application of higher dose of URP $\left(200 \% \mathrm{P}_{-} \mathrm{T}_{2}\right)$ recorded higher biomass yield than other treatments in $\mathrm{S}_{1}(\mathrm{pH}-4.15)$ and $\mathrm{S}_{2}$ (pH-5.03) whereas, URP+SSP $\left(\mathrm{T}_{3}\right)$ recorded maximum yield in $\mathrm{S}_{3}(\mathrm{pH}-5.82)$ might be due to dissolution of URP got slower with increased soil pH (Fig. 5).

Addition of URP+SSP or URP+ lime showed similar trend in $S_{1}$ and $S_{2}$ but lower than URP alone $\left(\mathrm{T}_{2}\right)$. However, URP+SSP combination recorded higher yield in $\mathrm{S}_{3}$ over all treatments. Combined application of URP+FYM or URP+ PSB recorded lower yield as compared to other treatments except control

The relative agronomic efficiency (RAE) of treatments was calculated taking URP+SSP $\left(\mathrm{T}_{3}\right)$ treatment as standard.

It was observed that sole application of higher dose of URP $\left(\mathrm{T}_{2}\right)$ recorded higher RAE than standard treatment in $S_{1}(107 \%)$ and $S_{2}$
(108\%), but the efficiency decreased in $\mathrm{S}_{3}$ (76\%) with increasing $\mathrm{pH}$. The efficiency of URP greatly influenced by soil $\mathrm{pH}$.

Correlations between soil available phosphorus, exchangeable calcium and hybrid napier grass yield

There were significant correlations between soil available phosphorus and biomass yield $\left(\mathrm{R}^{2}=0.964 * *\right)$ and soil exchangeable calcium and biomass yield $\left(\mathrm{R}^{2}=0.833^{* *}\right)$ (Fig. 6 and 7). The significant correlations indicate that the amounts of soil available $\mathrm{P}$ and exchangeable $\mathrm{Ca}$ derived during dissolution of rock phosphate could explain the yield variations. Phosphorus and calcium supplied to napier grass by amendments is consequently an important condition to achieve higher biomass yield in acid soils.

\section{Properties of soil and organic manure used}

Dissolution of phosphate rock is favoured by low $\mathrm{pH}, \mathrm{Ca}$ and $\mathrm{P}$ because such a situation provides protons and $\mathrm{Ca}$ and $\mathrm{P}$ sinks (Ranjan et al., 1996, Szilas 2002).The P component of $\mathrm{PR}$ dissolves in moist soil as per this following reaction $-\mathrm{Ca}_{10}\left(\mathrm{PO}_{4}\right)_{6} \mathrm{~F}_{2}$ $+12 \mathrm{H}^{+} \rightleftharpoons 10 \mathrm{Ca}^{2+}+6 \mathrm{H}_{2} \mathrm{PO}_{4}^{-}+2 \mathrm{~F}^{-}$

The dissolution rate therefore depends on the supply of $\mathrm{H}^{+}$ion (Kanabo and Gilkes, 1987a) and lowering of $\mathrm{Ca}^{2+}$ and $\mathrm{H}_{2} \mathrm{PO}_{4}{ }^{-}$ion activities through diffusion or adsorption reactions (Bolan and Hedley, 1989).The $\mathrm{pH}$ of the three soils used in our study was $\mathrm{S}_{1}-4.15, \mathrm{~S}_{2}-5.03$ and $\mathrm{S}_{3}-5.83$, which provides a conducive environment to denote $\mathrm{H}^{+}$ion for dissolution of RP. Bolan et al., (1986) and Tambunan (1988) reported that the $\mathrm{pH}$ of top $10 \mathrm{~cm}$ soils with $\mathrm{pH} 5$ to 6 were sufficient to dissolve 2.3 to $7.8 \mathrm{t}$ North Carolina PR per hectare under adequate moisture condition.

Lower available $\mathrm{P}(8.92-15.74 \mathrm{~kg} / \mathrm{ha})$ and exchangeable calcium $\left(1.32-1.50 \mathrm{c}\right.$ mol $\left(\mathrm{P}^{+}\right)$ $\mathrm{kg}^{-1}$ ) content of these soils provide a sink for 
dissolution of RP. Diffusion and adsorption of $\mathrm{P}$ on soil surface or by crop removal decrease the $\mathrm{P}$ concentration around $\mathrm{PR}$ particles and favour dissolution of PR (Kirk and Nye, 1986b, White 1988b, Kanabo and Gilkes 1987a). According to mass action law, PR dissolution releases $\mathrm{Ca}$ ion and soil with high Ca content would slow down PR dissolution (Hammand et al., 1986b). For many tropical acid soils, exchangeable $\mathrm{Ca}$ is relatively low, thus providing favourable condition for PR application. The nutrient composition of FYM $(\mathrm{N}, \mathrm{P}, \mathrm{K}$, and $\mathrm{Ca}$ ) makes it a fairly good amendment on acidic laterite soils.

\section{Effects of treatments on soil properties}

The increase in soil $\mathrm{pH}$ in all treatments except control can be attributed to the release of $\mathrm{Ca}$ due to dissolution of PR. Increase in $\mathrm{pH}$ and $\mathrm{Ca}$ has a positive impact on reduction of $\mathrm{P}$ sorption capacity and exchangeable $\mathrm{Al}$ in acid soils. Reduction of exchangeable Al was caused by formation of complex with $\mathrm{Ca}$. Combined addition of lime or SSP with URP maintained high $\mathrm{pH}$ during initial stage of growing period resulted in instant release of $\mathrm{Ca}$ from these sources over the period. On the otherhand, higher dose of URP released more $\mathrm{Ca}$ in latter stage of growth resulting higher $\mathrm{pH}$ than all other treatments. Hammond et al., (1986b) reported the high $\mathrm{pH}$ and increased calcium resulting from liming decrease PR dissolution. Therefore it is necessary to fix lime rates carefully to alleviate Al-toxicity problem, at the same time to avoid adverse effect of PR dissolution (Chien and Friesen, 1992). Mishra and Pattanaik (1997) working with acidic laterite soil observed the dissolution of different phosphate rocks reached equilibrium at 45 days of incubation due to build up of $\mathrm{Ca}$ and $\mathrm{P}$ ions released from PR due to inadequate size of sinks (in absence of a crop). However, under present study the dissolution of PR continued in long run since the nutrient removal by grass acted as a strong sink for calcium and phosphorus.

The decrease in exchangeable $\mathrm{Al}$ accompanied by increase in soil $\mathrm{pH}$ and exchangeable $\mathrm{Ca}$ has a positive effect on reducing $\mathrm{P}$ sorption capacity of soil (Sanyal and De Datta, 1991). This was revealed by an increase in available $P$ in all treatments except control upto $2^{\text {nd }}-5^{\text {th }}$ week and there after declined with decrease in soil $\mathrm{pH}$. The decline in soil available $\mathrm{P}$ with progress of time can partly be attributed to crop uptake which is continuous throughout all cuttings. Phosphorus adsorption, precipitation and lack of application of acidity ameliorating amendments could have been led to declining available $\mathrm{P}$ levels in the control treatments. Mokwunye et al., (1996) reported that $\mathrm{P}$ deficiency observed in acid soils is often associated with high $\mathrm{P}$ fixation between $\mathrm{pH}$ 5.0-6.0 where $\mathrm{H}_{2} \mathrm{PO}_{4}{ }^{-}$dominates (Furihata et al., 1992). Holford (1997) observed that more than $80 \%$ of applied $\mathrm{P}$ in acid soils undergoes adsorption, precipitation or conversion to the organic form. The higher levels of available $\mathrm{P}$ in URP+SSP treatment could be possible due to addition of water soluble $\mathrm{P}$ (through SSP) as well as consumption of $\mathrm{H}^{+}$from soil for dissolution of URP resulting decline in $\mathrm{P}$ fixation capacity of soil. Combined application of lime with URP increased soil $\mathrm{pH}$ and exchangeable $\mathrm{Ca}$ that reduces $\mathrm{P}$ fixation and increased available $P$. Dissolution of URP is not affected since crop uptake acted as a strong sink for $\mathrm{Ca}$ and $\mathrm{P}$. This was reflected in declining soil $\mathrm{pH}$ after $3^{\text {rd }}$ cutting. Higher level of available $\mathrm{P}$ in $\mathrm{T}_{2}$ $(200 \% \mathrm{P})$ after $5^{\text {th }}$ cutting was associated with decline in soil $\mathrm{pH}$ that favour dissolution of ' $\mathrm{P}$ ' in latter stage of growth.

FYM combined with URP also increases available $\mathrm{P}$ in soil through chelation and decomposition. The decomposition products of organic materials have significant chelation capacity that lowers the activity of $\mathrm{Fe}$ and $\mathrm{Al}$ 
which form insoluble salts with $\mathrm{P}$ and so liberate phosphorus. Several authors have reported competition between organic acids and $\mathrm{P}$ for sorption sites that usually favours adsorption of organic acids and delays $\mathrm{P}$ adsorption (Volante and Gianfreda 1993, Geelhoed et al., 1999).

Inclusion of PSB with URP also recorded similar effect on soil $\mathrm{pH}$ and available P. PSB application enhances dissolution of phosphate rock through production of organic acid and chelating substances (Adhya et al., 2015) as well as production of growth promoting hormones.

\section{Biomass yield and Phosphorus use efficiencies of hybrid napier grass}

The lower biomass yield of hybrid napier in control may be attributed to the low availability of $\mathrm{P}$ due to fixation in acid soil. Conversely, the supply of $\mathrm{P}$ and $\mathrm{Ca}$ by $\mathrm{P}$ sources (URP, SSP) and amendments (lime, FYM, PSB) in combination contributed significantly higher biomass yield. Mishra and Pattanaik (1997) reported similar result with hybrid napier grass in an acidic sandy loam soil ( $\mathrm{pH} 5.6$, low available P). Subehia and Minas (1993) studied the effect of URP with FYM and poultry manure in clay loam soil with pH 5.7.

The organic manure enhanced the dissolution of PR or chelation of $\mathrm{Ca}^{2+}$ ions and subsequently lowering of $\mathrm{Ca}^{2+}$ ion activity in soil solution providing a sink for $\mathrm{Ca}^{2+}$.

Under certain field conditions such as high $\mathrm{pH}$, short term crop or low reactive PR, the agronomic efficiency of PR may not be feasible as that of water soluble SSP. Mixing of PR with SSP can be effective under such situation. In this study URP and SSP mixture in 1:1 ratio recorded higher biomass yield in $\mathrm{S}_{3}$ (soil $\mathrm{pH}$ 5.82) than URP +lime or lone URP treatment but it was inferior to these two treatment in $\mathrm{S}_{1}$ (soil $\left.\mathrm{pH} 4.15\right)$ and $\mathrm{S}_{2}$ (soil $\mathrm{pH}$ 5.08) (Fig. 4).

Similar observation was made by Prochnow et al., (2004) in Brazil for wheat and rye grass with PR: SSP compaction at 1:1 ratio because the water soluble SSP provides $\mathrm{P}$ to plants initially (Starter effect) resulting in better plant root development, which in turn allowed the plants to utilize the PR more effectively in later stage. Such a mixtures further reduces the $\mathrm{P}$ fixation by depressing the activity of free $\mathrm{Fe}$ and $\mathrm{Al}$ in soil solution and enhance the solubility of PR by action of initial soil acidity created in root rhizosphere (Mc Lean and Wheeler, 1964).

Higher dose of URP (200\% P) application in $\mathrm{S}_{1}$ (soil $\mathrm{pH}$ - 4.15) and $\mathrm{S}_{2}$ (soil $\mathrm{pH}$-5.08) recorded higher biomass yield than URP+SSP and URP+ lime treatments because of higher dissolution of URP due to low $\mathrm{pH}$, low available $\mathrm{P}$ and low exchangeable $\mathrm{Ca}$ content in soil during entire crop period. However, this treatment in $\mathrm{S}_{3}$ with high $\mathrm{pH}$ and high $\mathrm{Ca}$ content was inferior to URP + SSP or URP + lime due to slow down of PR dissolution, according to mass action law. (Hammond et al., 1986 b).

In conclusion, phosphate rock is a viable alternative to the expensive water soluble $\mathrm{P}$ fertilizers (SSP) in increasing crop productivity in acid soils of India. Application of URP alone or with amendments increased soil $\mathrm{pH}$, available $\mathrm{P}, \mathrm{Ca}$, biomass yield of hybrid napier grass. The effect was higher when applied with lime or mixed with SSP in 1:1 ratio. Higher dose of URP alone was as effective as URP: SSP mixture in 1:1 ratio for long duration crops as reflected by biomass yield and RAE and can therefore be used as an affordable alternative to the more expensive water soluble SSP fertilizer. Effect of FYM or PSB on URP dissolution rate as reflected by soil available $\mathrm{P}$, soil $\mathrm{pH}$, exchangeable $\mathrm{Ca}$ and biomass yield of hybrid 
napier was low as compared to combined application of URP+ SSP mixture or URP+ lime.

\section{References}

AOAC (1995) Official Method of Analysis. Association of official Agricultural Chemists. $12^{\text {th }}$ Ed. Washington, D.C.

Adhya, T.K., Reddy, Kumar, N.,.N., Reddy, G., Pondile, A.P., Bee, H. and Samantaray, B. (2015) Microbial mobilization of soil Phosphorus and sustainable $\mathrm{P}$ management in agricultural soils. Current Science 108(7): 1280-1287.

Barrow, N.J. (1983) On the reversibility of phosphate sorption by soils. Journal of Soil Science 34:751-758.

Bolan, N.S., and Hedley, M.J. (1989) Dissolution of phosphate rocks in soils. I. Evalution of extraction methods for the measurement of phosphate rock dissolution. Fert.Res. 19: $65-75$.

Bolan, N.S., Syers, J.K., and Tilman, R.W. (1986) Ionic strength effects on surface charge and adsorption of phosphate and sulphate on soils. J.Soil Sci. 37: 379-388.

Bouyoucos, G.J. (1962) Hydrometer method improved for making particle size analysis of soils. Agron. J. 54:464465.

Bray,R.H., and Kurtz, L.T. (1945) Determination of total organic and available forms of phosphorus in soils. Soil Sci. 59:39-45.

Chien, S.H., and Friesen, D.K. (1992) Phosphate rock for direct application. In: Workshop on Future Directions for Agricultural Phosphorus Research 47 52. TVA Bulletin Y-224, Muscle Shoals, AL, USA.

Das, P.K., Mishra, U.K., and Sahu, S.K. (1990) Evaluation of the direct effect of Udaipur rock phosphates on maize in the acid lateritic soils of Orissa. Orissa Journal Agricultural Research, (3): 109-114.

Dash, R.N., Mohanty, S.K., and Pattanaik, S. (1988) Influence of reactivity of phosphate rocks on $\mathrm{P}$ Solubilization by dhaincha (Serbania aculeata). J. Indian Soc. Soil Sci., 36:375-378.

Dwibedi, G.K., and Dwibedi, M. (1990) Relative efficiency of Mussorie rock phosphate, single super phosphate and their mixture in acid soil under lentilmaize crop sequence. Annals of Agricultural Research. 11: 28-38.

Furihata, T., Suzuki, M. and Sakurai, H. (1992) Kinetic characterization of two phosphate uptake systems with different affinities in suspensioncultured Catharanthus roseus protoplasts. Plant Cell Physiol. 33: $1151-1157$.

Geelhoed, J.S., Van Riem Sdijk, W.H., Findenegg, G.R. (1999) Simulation of the effect of citrate exudation from roots on the plant availability of Phosphate adsorbed on goethite. Eur. J. Soil Sci. 50: 379-390.

Gupta, P.K. (2007) Determination of exchangeable Calcium and Magnesium. Soil, Plant, Water and Fertilizer analysis AGROBIOS (INDIA), $2^{\text {nd }}$ edition: 2007 page 9596. ISBN No. 81-7754-306-7.

Hammond, L.L., Chien, S.H., and Mokwunye, A.U. (1986b) Agronomic Value of unacidulated and parially acidulated phosphate rocks indigenous to the tropics. Adv. in Agron. 40: 89140.

Hanway, J.J., and Heidal. (1952) Soil analysis methods as used in Lowa state. College of Soil Testing Laboratary, Lowa. Agric., 57: 1-31.

Hedley,M. and Mclaughlin, M. (2005) Reaction of phosphate fertilizer and by-products in soils. In: Sims.JT, 
Sharpley.AN(ed). Phosphorous: agriculture and environment, Agronomy Monograph No.46,ASACSSA-SSSA, Madison, p. 181-252.

Holford, J.C.R. (1997) Soil phosphorus: its measurement, and its uptake by plants. Aust. J. Soil Res., 35: 227-239.

Hughes, J.C., and Gilkes, R.J. (1994) Rock phosphate dissolution and bicarbonate soluble $\mathrm{P}$ in some soils from southwestern Australia. Australian Journal of Soil Research 32:767-779.

Jackson, M.L. (1973) soil Chemical Analysis. Prentice Hall of India Pvt. Ltd. New Delhi.

Kanabo, I.A.K., Gilkes, R.J. (1987a) The role of soil $\mathrm{pH}$ dissolution of phosphate rock fertilizers. Fertilizers and Research 12: 165-179.

Kirk, G.J.D., and Nye P.H. (1986b) A simple model for predicting the rates of dissolution of sparingly soluble calcium phosphates in soil. II. Application of the model. J. Soil Sci., 37: 541-55.

McLean, E.O., Aluminium, In: Black, C.A. (Ed.). (1965) Methods of soil analysis: Part-2. Chemical methods. Madison: ASA, p-978-998.

McLean, E.O. and Wheeler, R.W. 1964. Partially acidulated rock phosphate as a source of phosphorus to plants: I. Growth chamber studies. Soil Sci. Soc. Am. Proc. 28: 545-550.

Mishra, U.K., and Pattanaik, S.K. (1997) Characterization of rock phosphate for direct use of different cropping sequenses. Technical report of the USIndia Fund project number: In-AES708, Grant number FG-In-744, 19911995.

Mitra, G.N., and Mishra, U.K.,. (1991) Evaluation of Udaipur rock phosphatic fertilizers in the soils of Orissa. Research Bulletin 1/91, OUAT.

Mokwunye, A.U., De Jager, A. and Smaling,
E.M.A. (1996) Restoring and maintaining the productivity of West African soils: key to sustainable development. IFDC-Africa, LEI-DLO and SC-DLO.

Pattanaik, S. (1988) Reactivity of Indian phosphate rock in relation to crystal chemical structure of their apatite. J. Indian Soc. Soil Sci. 36:619-635.

Prochnow, L.I., Chien, S.H., Carmona, G., Henao, J. (2004) Greenhouse evaluation of two phosphorus sources produced from a Brazilian phosphate rock. Agron. J. 96:761-768.

Ranjan, S.S.S., Watkinson, J.H., Sinclair, A.G. (1996) Phosphate rocks for direct application to soils. Advances in agronomy, 57. p.77-159.

Sanyal, H. and Saha, S.K. (1988) Proceeding of the seminar on use of rock phosphate in neutral soils, T.N.A.U., Coimbatore, pp 84-93.

Sanyal, S.K., and De Datta S.K. (1991) Chemistry of phosphorus transformations in soil. In Stewart, B.A., Adv. Soil Sci. 16: 31-60.

Schollenger,C.J., and Simon,R.H. (1945) Determination of exchange capacity and exchangeable bases in soil. Ammonium acetate method. Soil Science, 59: 13-24.

Subbiah, B.V., and Asija, G.L. (1956) A rapid procedure for determination of available Nitrogen in soils. Current Science, 25:259-260.

Subehia, S.K., and Minhas, R.S., (1993) Phosphorus availability from Udaipur rock phosphate as influenced by different Organic amendments. J. Indian Soc. Sci. 41:96-99.

Syers, J.K., Johnston, A.E., Curtin, D. (Eds) (2008) Efficiency of soil and fertilizer phosphorus use. Reconciling changing concepts of soil phosphorus behaviour with agronomic information. FAO Fertilizer and Plant Nutrition Bulletin 
(Food and Agriculture Organisation of the United Nations: Rome, Italy).

Szilas, C. (2002) The Tanzanian Minjingu phosphate rock -possibilities and limitations for direct application. Ph.D. Thesis, Royal Veterinary and Agricultural University, Copenhagen.

Tambunan, D. (1988) “ A laboratory assessment of the $\mathrm{pH}$ buffering capacity and lime requirements of selected New Zealand soils.” Dip. of Agric. Sci.,Massey University, Palmerston North, New Zealand.

Walkley, A., and Black, I.A. (1934) An examination of Degtjare method for determining soil organic matter and a proposed modification of the Chromic acid titration method. Soil Sci. 37:2937.

Wheeler, D.M., and Edmeades, D.C. (1964)
Measuring the lime requirements of New Zealand soils. In." Lime in Agriculture (Eds B.L.J. Jackson and D.C. Edmeades). pp 63-4.

White, R.E. (1988b) Soil-Plant-Fertilizer interaction: New developments involving phosphate. New Zealand Agricultural Science. 22:58-62.

Woodruff, C.M. (1948) Testing soils for lime requirement by means of a buffered solution and the glass electrode. Soil Sci. 66:53-63.

Volante, A. and Gianfreda, L.(1993) Competition in adsorption between phosphate and Oxalate on an aluminium hydroxide montmorillonite complex. Soil Sci. Soc. Am. J. 57, 1235- 1241 .

\section{How to cite this article:}

Debasis Sarangi, Dinabandhu Jena and Kabita Mishra. 2019. Relative Efficiency of Udaipur Rock Phosphate Combined with Amendments in Acid Soils of Odisha, India. Int.J.Curr.Microbiol.App.Sci. 8(01): 322-341. doi: https://doi.org/10.20546/ijcmas.2019.801.034 